\title{
Misorientation dependence of zinc incorporation in GaAs
}

\author{
M.J. Anders *, P.R. Hageman, L.J. Giling \\ Department of Experimental Solid State Physics, Research Institute for Materials, Faculty of Science, University of Nijmegen, \\ Toernooineld, 6525 ED Nijmegen, Netherlands
}

Received 8 December 1993; manuscript received in final form 16 June 1994

\begin{abstract}
Doping studies of zinc have been carried out for metalorganic chemical vapour deposition (MOCVD) grown GaAs, with diethyl zinc as precursor. The zinc incorporation has been studicd as a function of the zinc input mole fraction, growth temperature and substrate misorientation. It is found that the zinc incorporation on (001) substrates misoriented towards [011] and [011] directions showed a consistent and similar dependence on the misorientation angle at various zinc input mole fractions, with an observed minimum in the $\mathrm{Zn}$-incorporation at $4^{\circ}$ off. The observed temperature dependence leads to a apparent activation energy of $-59 \mathrm{kcal} / \mathrm{mol}$ for zinc incorporation which is attributed to the enthalpy involved in the formation of two bonds during the incorporation. The dependence on the misorientation angle is explained by competition between the influence of "step trapping" at lower misorientation (and higher step velocities) and the increase of available kinksites at higher misorientation angles.
\end{abstract}

\section{Introduction}

The most widely applied p-dopant in MOCVD grown (Al)GaAs nowadays is diethylzinc (DEZn). Although numerous incorporation studies are known from the literature [1-13], none of them was directed at the misorientation dependence. Prompted by previous work on indium and silicon incorporation $[14,15]$ we present in this article the experimental results on zinc doping of GaAs as a function of the DEZn input mole fraction, growth temperature and substrate orientation. We compare our results with a previous paper [16] and try to show whether an adsorption/desorption equi-

\footnotetext{
* Corresponding author
}

librium can account for the observed incorporation behaviour, as was stated in that paper.

\section{Experimental procedure}

The experiments were carried out in a commercially available, computer controlled MOCVD reactor. The reactor can be operated at low pressures, is infrared heated and the horizontal liner in the cylindrical quartz tube has a rectangular cross section. The top of the cell is not controllably cooled, which implies that only the bottom temperature of the cell is known and that the temperature of the top of the cell is floating.

The growth was performed with arsine $\left(\mathrm{AsH}_{3}\right)$ and trimethyl gallium (TMG) as growth precursors, and hydrogen, purified by a Pd-diffusion 
cell, as carrier gas. Diethyl zinc (DEZn) was used as precursor for the zinc doping.

All experiments were performed at $20 \mathrm{mbar}$ and constant $P_{\mathrm{TMG}}$ and $P_{\mathrm{AsH}_{3}}(\mathrm{~V} / \mathrm{III}$ ratio of $125)$ at growth temperatures ranging from 560 to $720^{\circ} \mathrm{C}$. The mean gas flow rate in the reactor was $228 \mathrm{~cm} / \mathrm{s}$. The growth rate varied between 0.02 and $0.04 \mu \mathrm{m} / \mathrm{min}$ dependent on the growth temperature.

The GaAs substrates were all semi-insulating and chemo-mechanically polished on one side with the following orientations: (100) $6^{\circ}(0 \overline{1} 1)$, (100) $6^{\circ}(011),(100) 4^{\circ}(0 \overline{1} 1),(100) 4^{\circ}(011),(100)$ $2^{\circ}(0 \overline{1} 1),(100) 2^{\circ}(011)$ and $(100)$.

After cleaving and staining the thickness of the grown layers was measured by interference-contrast microscopy or, if necessary, by scanning electron microscopy (SEM). The samples were electrically characterized by Hall-Van der Pauw measurements performed at room temperature. A clover leaf configuration was used.

\section{Results}

At all growth conditions the epitaxial layers showed mirror-like surfaces, independent of the diethyl zinc input mole fraction, growth temperature and substrate orientation. As all experiments were carried out at conditions where no saturation of the charge carrier density $(p)$ is observed, $p$ can be taken equal to the density of incorporated zinc. The background concentrations for undoped material were at level of $n \approx 5 \times 10^{14}$ $\mathrm{cm}^{-3}$, i.e. insignificant compared to the charge carrier densities that we measured.

In order to study the temperature dependence of the zinc incorporation for the different substrate orientations, a series of experiments was performed at temperatures ranging from 560 to $720^{\circ} \mathrm{C}$ under constant growth conditions and with a DEZn mole fraction of $3.5 \times 10^{-5}$. The results are shown in Fig. 1, in which the natural log of the hole concentration $p$ as obtained from Hall measurements is plotted versus the reciprocal temperature.

Fig. 1 shows that there is no significant difference in apparent activation energy for the incor-

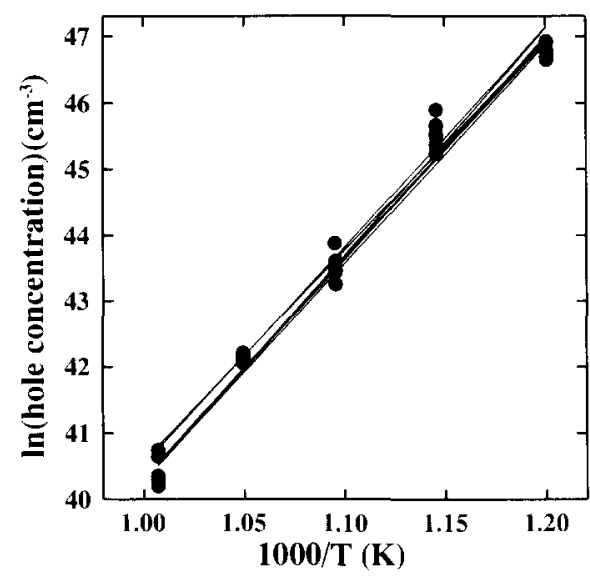

Fig. 1. Arrhenius plot of zinc incorporation on (100) planes offset to different directions.

poration of zinc for the different misorientations. The measured apparent activation energy $\left(E_{\text {app }}\right)$ is $-59 \pm 3 \mathrm{kcal} \mathrm{mol}^{-1}$. This negative value represents an energy release, as for instance in bond formation. The observed value is within experimental error equal to the value obtained by Hageman et al. [17]; experiments that were carried out under the same conditions but at lower input concentrations DEZn. The equal value of the $E_{\text {app }}$ for the differently misoriented substrates implies that the incorporation mechanism is the same for all orientations. According to Ref. [17] the adsorption/desorption equilibrium of the zinc species, most probably at a step, plays an important role during the incorporation of zinc.

For a series of experiments the zinc incorporation, at a fixed input mole fraction of the dopant, was studied as function of the misorientation angle between $0^{\circ}$ and $6^{\circ}$ for misorientations towards (011) and $(0 \overline{1} 1)$. Fig. 2 shows semi logarithmic plots of the hole concentration versus the misorientation angle for misorientations towards (011) and $(0 \overline{1} 1)$, at a growth temperature of $720^{\circ} \mathrm{C}$, at an input mole fractions DEZn of $3.5 \times 10^{-6}$. (To monitor any possible influence of saturation, these experiments were repeated for DEZn fractions up to $2.7 \times 10^{-4}$. No noticeable different effect was observed for the different zinc input mole fractions.) The reproducibility of the data points was checked by duplo experiments. No 


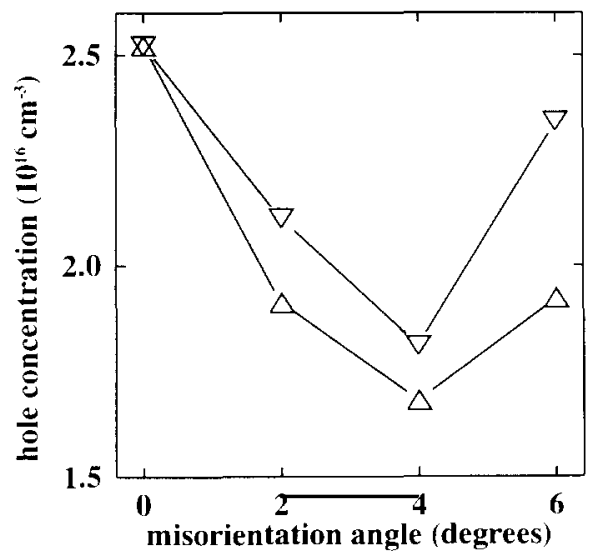

Fig. 2. Hole concentration versus misorientation angle: $(\nabla)$ towards $(001),(\Delta)$ towards $(0 \overline{1} 1)$.

significant deviations were observed between these duplo experiments, all deviations being much smaller than the differences noted in the trend.

From this figure we see that:

- the exact orientation always exhibits a higher zinc incorporation rate than misoriented substrates;

- a minimum is observed at $4^{\circ}$ off;

- the dependence on the misorientation angle is the same for misorientations in the direction of the $(0 \overline{1} 1)$ plane and the $(011)$ plane.

The observed overall behaviour of both misorientation directions is the same which is a clear

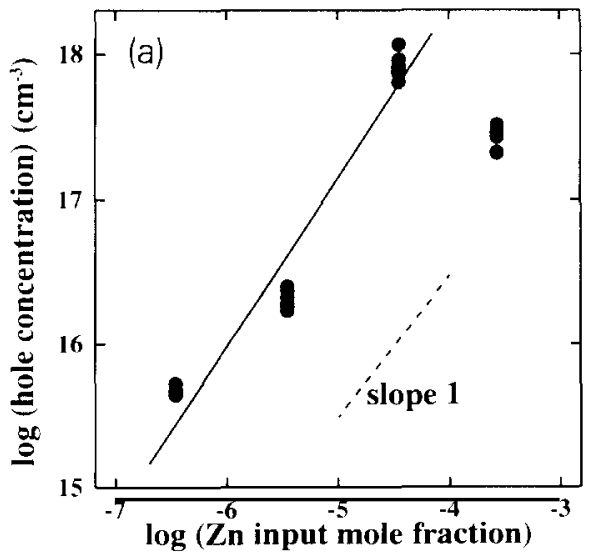

indication that step properties are involved. The offset which still exists between the two misorientation directions of Fig. 2 demonstrates that part of the incorporation is also determined by the nature of the steps.

\section{Discussion}

At the temperatures involved in these experiments, DEZn is assumed to be completely dissociated to atomic zinc. Therefore the incorporation process can be described by the following equilibrium processes:

(a) Adsorption/desorption of the zinc species on a free surface site $\left(*_{\text {surf }}\right)$ :

$\mathrm{Zn}_{\text {gas }}+*_{\text {surf }} \stackrel{k_{\text {ads }}}{\underset{k_{\text {des }}}{\rightleftharpoons}} \mathrm{Zn}_{\text {surf }}^{*}$,

$K_{\mathrm{ads}}=\frac{k_{\mathrm{ads}}}{k_{\mathrm{des}}}=\frac{\left[\mathrm{Zn}_{\mathrm{surf}}^{*}\right]}{\left[\mathrm{Zn}_{\mathrm{gass}}\right]\left[*_{\text {surf }}\right]}, \quad \Delta H=\Delta H_{\mathrm{ads}}$

(b) diffusion followed by adsorption/desorption at a free step site $\left(*_{\text {step }}\right)$ :

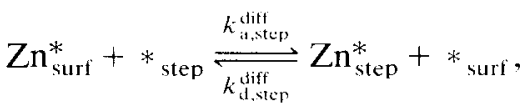

$$
\begin{aligned}
& K_{\text {step }}=\frac{k_{\mathrm{a}, \text { step }}^{\text {diff }}}{k_{\mathrm{d}, \text { step }}^{\text {diff }}}=\frac{\left[\mathrm{Zn}_{\text {step }}^{*}\left[*_{\text {surf }}^{*}\right]\right.}{\left[\mathrm{Zn}_{\text {surf }}^{*}\right]\left[*_{\text {step }}\right]}, \quad \Delta H=0 ;
\end{aligned}
$$

Fig. 3. (a) Log-log plot of hole concentration versus input mole fraction. (b) Behaviour at lower $T$. 
(c) then diffusion followed by adsorption/desorption at a kink site $\left(*_{\text {kink }}\right)$ :

$$
\begin{aligned}
& \mathrm{Zn}_{\text {step }}^{*}+*_{\text {kink }} \underset{k_{\mathrm{d}, \mathrm{kink}}}{\stackrel{k_{\mathrm{a}, \mathrm{kink}}}{\rightleftharpoons}} \mathrm{Zn}_{\mathrm{kink}}^{*}+*_{\text {step }} \text {, } \\
& K_{\text {kink }}=\frac{k_{\text {a,kink }}}{k_{\mathrm{d}, \text { kink }}}=\frac{\left[\underline{\mathrm{Zn}}_{\text {kink }}^{*}\right]\left[*_{\text {step }}\right]}{\left[\mathrm{Zn}_{\text {step }}^{*}\right]\left[*_{\text {kink }}\right]}, \\
& \Delta H=\Delta H_{\mathrm{ads}} \text {; }
\end{aligned}
$$

(d) and finally incorporation into the bulk:

$$
\mathrm{Zn}_{\text {kink }}^{*} \longrightarrow \mathrm{Zn}_{\text {bulk }} \text {. }
$$

It is thought that the overall $\left[\mathrm{Zn}_{\text {kink }}^{*}\right]$ equilibrium has in part a kinetic character because of what in an earlier paper [17] is called trapping of $\mathrm{Zn}$ by the moving steps, but what actually is the decoupling of the $\mathrm{Zn}$ equilibrium in the surface layer from that of the $\mathrm{Zn}$ in the bulk. The mechanism responsible for this decoupling is the higher growth velocity of GaAs normal to the surface compared to the speed of outdiffusion of $\mathrm{Zn}$ from the bulk to the surface.

It should be noted that this kind of trapping manifests itself quite conclusively in the log-log plot of the incorporation of dopant species versus input partial pressure of the dopant. Whenever trapping occurs this should lead to a slope of 1 [18]. This check was performed for this study as well. In Fig. 3a the hole concentration is plotted versus the input mole fraction DEZn for the different substrate misorientations at a growth temperature of $720^{\circ} \mathrm{C}$. (Fig. $3 \mathrm{~b}$ shows the behaviour at a lower temperature $\left(640^{\circ} \mathrm{C}\right)$, i.e. at higher bulk concentrations of zinc) In the log-log plot indeed a linear behaviour with a slope of 1 is obtained for each orientation at the lower input values (which are used in this study). For higher $P_{\mathrm{DEZn}}$ the incorporation saturates. This firmly supports the assumption that the zinc incorporation is governed by the adsorption/desorption equilibrium of the zinc species followed by incorporation through "step trapping".

We can calculate the equilibrium concentration of $\mathrm{Zn}_{\text {kink }}^{*}$ from the equilibrium equations above, which leads to:

$$
\left[\mathrm{Zn}_{\text {kink }}^{*}\right]=K_{\text {kink }} K_{\text {step }} K_{\text {ads }}\left[*_{\text {kink }}\right]\left[\mathrm{Zn}_{\text {gas }}\right] \text {. }
$$

The observed temperature dependence of the zinc incorporation follows directly from the temperature dependence of the equilibrium constants in the previous equation (viz. $2 \Delta H$ ). As $\left[\mathrm{Zn}_{\text {gas }}\right]$ is linearly proportional to $\left[\mathrm{Zn}_{\text {input }}\right]$, the equilibrium concentration of zinc at a kink site is directly proportional to the input concentration of zinc and to the concentration of kinks. In a simple picture this equilibrium concentration will be covered by GaAs layers when the outdiffusion of trapped $\mathrm{Zn}$ in the bulk of the crystal towards the surface is slower than the growth rate of GaAs normal to the surface, i.e. $r_{\text {growth }}>$ $D_{\text {outdiff }} / \lambda_{\mathrm{b}}$, where $\lambda_{\mathrm{b}}$ is a characteristic diffusion length of $\mathrm{Zn}$ in the bulk which typically is in the order of a few lattice units. For the growth rates encountered in this study viz. 0.02-0.04 $\mu \mathrm{m} / \mathrm{min}$ we calculate $D_{\text {outdiff }}<2.5 \times 10^{-13} \mathrm{~cm}^{2} / \mathrm{s}$. Literature values for the bulk diffusion of zinc in GaAs vary, dependent on the III $/ \mathrm{V}$ ratio, between $10^{-12}$ and $10^{-14} \mathrm{~cm}^{2} / \mathrm{s}[19,20]$. The uncertainty in these values is large enough to make the trapping condition also on this basis acceptable.

In order to check whether perhaps we can explain the results of Fig. 2 in terms of $\mathrm{Zn}$ and $\mathrm{Ga}$ fluxes to the step instead of step trapping, we proceed as follows. From the continuity equation for $\mathrm{Ga}$ and $\mathrm{Zn}$ (see also Ref. [21]), taking into account that the surface concentration for the adsorbed zinc species close to the step might not be zero, we obtain:

$$
\begin{aligned}
& r(\mathrm{Zn})= 2 k_{\mathrm{a}_{\mathrm{Zn}}} c_{\mathrm{Zn}}^{0} \lambda_{\mathrm{Zn}} \\
& \times \frac{\sinh \frac{y_{0}}{2 \lambda_{\mathrm{Zn}}}}{\cosh \frac{y_{0}}{2 \lambda_{\mathrm{Zn}}}+\frac{\sqrt{k_{\mathrm{d}_{\mathrm{Zn}}} D_{\mathrm{s}}}}{k_{\mathrm{inc}_{\mathrm{Zn}}}} \sinh \frac{y_{0}}{2 \lambda_{\mathrm{Zn}}}} .
\end{aligned}
$$

Likewise we obtain for gallium:

$r(\mathrm{Ga})=2 k_{\mathrm{a}_{\mathrm{Ga}}} c_{\mathrm{Ga}}^{0} \lambda_{\mathrm{Ga}} \tanh \frac{y_{0}}{2 \lambda_{\mathrm{Ga}}}$.

Where the surface concentration of adsorbed Ga close to the step now has been taken to be zero, which is true for the growth of GaAs in the gas phase diffusion limited regime. In these equations $y_{0}$ is the width of the terrace, $k_{\mathrm{a}}$ and $k_{\mathrm{d}}$ are 
the rate constants for the adsorption and desorption from the gas phase, $k_{\text {inc }}$ is the rate constant for incorporation at the step, $c^{0}$ the concentration in the middle of the terrace, $\lambda$ the surface diffusion length and $D_{\mathrm{s}}$ the surface diffusion coefficient.

The flux ratio $r(\mathrm{Zn}) / r(\mathrm{Ga})$ is a function of $y_{11}$, i.c. the misorientation of the substrates, and of the surface diffusion lengths $\lambda_{\mathrm{Ga} Z \mathrm{Zn}}$ of the Ga and $\mathrm{Zn}$ species. $\lambda_{\mathrm{Ga}}$ and $\lambda_{\mathrm{Zn}}$ can be calulated fom the formula $\lambda=D_{\mathrm{s}} / k_{\mathrm{d}}$, where $D_{\mathrm{s}}=\frac{1}{4} \nu l^{2} \mathrm{e}^{-E_{\mathrm{m}} / k T}$ and $k_{\mathrm{d}}=\nu \mathrm{e}^{-E_{\mathrm{h}} / k T}, \nu=10^{1.3} \mathrm{~s}^{-1}, l=3.8 \AA$ and $E_{\mathrm{m}}$ is half the binding energy $E_{\mathrm{b}}$. Using $E_{\mathrm{b}}(\mathrm{Zn})$ $=29.5 \mathrm{kcal} / \mathrm{mol}$ (see later) and $E_{\mathrm{b}}(\mathrm{Ga})=39.4$ $\mathrm{kcal} / \mathrm{mol}$, we calculate $\lambda_{\mathrm{Ga}}=270 \AA$ and $\lambda_{\mathrm{Zn}}=78$ A.

For misorientation angles between $2^{\circ}$ and $6^{\circ}$ (see Fig. 2), $y_{0}$ varies between 28 and $86 \AA$. For this situation we always have $\lambda_{\mathrm{Ga}}>\lambda_{\mathrm{Zn}}>1 / 2 y_{0}$. It can be shown that because of this, the flux ratio $r(\mathrm{Zn}) / r(\mathrm{Ga})$ will increase with increasing misorientation angle. This means that the negative slope as obtained in Fig. 2 between $0^{\circ}$ and $4^{\circ}$ cannot be explained on the basis of fluxes to the step.

As argued before we have evidence that the negative slope can be accounted for by step trapping, the high $\mathrm{Zn}$ concentration at $0^{\circ}$ and $2^{\circ}$ being the result of the fast moving surface steps. A similar phenomenon is observed in the incorporation of dopants during growth from the melt or from solution.

It is well known that surface (step) concentrations in common cases are higher than bulk concentrations $\left(k_{\mathrm{eff}}<1\right)$. This is the reason why at too high growth rates higher dopant concentrations are incorporated. The same phenomenon applies in our case, only here the role is taken over by the fast moving steps, i.e. the step trapping mechanism.

The temperature dependence of the zinc incorporation is determined by the temperature behaviour of the equilibrium concentration of $\mathrm{Zn}$ at the kinks as given in Eq. (1), i.e. by the temperature dependence of the product of the three equilibrium constants $K_{\text {ads }} K_{\text {step }} K_{\text {kink }}$. In essence this product contains the formation of two $\mathrm{Zn}-\mathrm{As}$ bonds. The $-59 \mathrm{kcal} / \mathrm{mol}$ found for $E_{\text {app }}$ can now be associated with an adsorption enthalpy of two $\mathrm{Zn}-\mathrm{As}$ bonds; one formed when the zinc species adsorbs on the surface, the second one formed on incorporation at a kink site.

The clear pattern exhibited by the zinc incorporation as a function of the offset towards $(0 \overline{1} 1)$ or (011) now is explained as follows: due to the adsorption/desorption equilibrium the mean residence time at the surface of the zinc species is constant at constant growth conditions. However, since higher offset angles lead to lower step velocities (at a constant growth rate), the probability that a zinc species is caught by a moving step will be diminished, i.e. there is less incorporation through step trapping.

On the other hand, at higher step densities also the number of kink sites increases, leading to a larger concentration of zinc at kink positions (see Eq. (1)), and so to a higher concentration in the bulk. This competitive behaviour explains the observed minimum in the zinc incorporation.

\section{Conclusions}

Misorientation dependent zinc incorporation studies were performed for the growth of GaAs on (100) and vicinal surfaces. It was shown that the degree of misorientation had no influence on the heat of incorporation. This is consistent with an adsorption/desorption equilibrium of the zinc species at the surface followed by incorporation through "step trapping".

Surfaces misoriented towards $(0 \overline{1} 1)$ or $(011)$ showed a clear minimum in the zinc incorporation rate at $4^{\circ}$, a phenomenon that can be explained by a balance between the step velocity and the number of kink sites.

\section{Acknowledgments}

The authors are greatly indebted to G.J. Bauhuis for performing the electrical characterization of the samples. This work was financed by the "Stichting Scheikundig Onderzoek in Nederland" (SON), Project No. 332-012 and by the "Nederlandse Maatschappij voor Energie en Milieu" (NOVEM), Project No. 41.220-003.1. 


\section{References}

[1] R.W. Glew, J. Crystal Growth 68 (1984) 44.

[2] Y.K. Su, C.Y. Chang, T.S. Wu, Y.C. Chou and C.Y. Nee, J. Crystal Growth 67 (1984) 472.

[3] K. Okamoto, H. Mawatari, K. Yamaguchi and A. Noguchi, J. Crystal Growth 98 (1989) 630.

[4] S.Z. Sun, E.A. Armour, K. Zheng and C.F. Schaus, J. Crystal Growth 113 (1991) 103.

[5] M.A. Tischler, R.M. Potemski, T.F. Kuech, F. Cardone, M.S. Goorsky and G. Scilla, J. Crystal Growth 107 (1991) 268.

[6] T.F. Kuech, P.-J. Wang, M.A. Tischler, R. Potemski, G.J. Scilla and F. Cardone, J. Crystal Growth 93 (1988) 624.

[7] P. Enquist, J.A. Hutchby and T.J. de Lyon, J. Appl. Phys. 63 (1988) 4485.

[8] G. Keil, M. le Metayer, A. Cuquel and D. le Pollotec, Rev. Physique Appl. 17 (1982) 405.

[9] J.P. Hallais, Acta Electron. 21 (1978) 129.

[10] V. Aebi, C.B. Cooper III, R.L. Moon and R.R. Saxena, J. Crystal Growth 55 (1981) 517.

[11] H.M. Manasavit and A.C. Thorsen, J. Electrochem. Soc. 119 (1972) 99.
[12] G. Vassilieff and B. Saint-Cricq, J. Appl. Phys. 54 (1983) 4581.

[13] P.J. Wright, B. Cockayne, A.C. Jones and E.D. Orrell, J. Crystal Growth 91 (1988) 63.

[14] X. Tang, J. te Nijenhuis, Y. Li and L.J. Giling, J. Crystal Growth 107 (1991) 263.

[15] J. te Nijenhuis, Thesis, Nijmegen (1993).

[16] P.R. Hageman, M.H.J.M. de Croon, J.N.H. Reek and L.J. Giling, J. Crystal Growth 116 (1992) 169.

[17] P.R. Hageman, M.H.J.M. de Croon, X. Tang and L.J. Giling, J. Crystal Growth 129 (1993) 281.

[18] A.A. Chernov, Modern Crystallography, III, Crystal Growth, Springer Series in Solid State Sciences 36 (Springer, Berlin, 1984) p. 159.

[19] N.H. Ky, L. Pavesi, D. Araújo, J.D. Ganière and F.K. Reinhart, J. Appl. Phys. 69 (1991) 7585.

[20] C.H. Ting and G.L. Pearson, J. Electrochem. Soc. 118 (1971) 1454.

[21] W.K. Burton, N. Cabrera and F.C. Frank, Phil. Trans. Roy. Soy. London A 243 (1951) 299.

[22] M. Tanaka, T. Suzuki and T. Nishinawa, J. Crystal Growth 111 (1991) 168. 